MÖBUS, C. \& EILERS, M., Mixture of Behaviors and Levels-of-Expertise in a Bayesian Autonomous Driver Model (paper accepted), 1st Intern. Conf. On Applied Digital Human Modeling, 17-20 July, 2010, Intercontinental, Miami Florida, USA

\title{
Mixture-of-Behaviors and Levels-of-Expertise in a Bayesian Autonomous Driver Model
}

\author{
Claus Möbus ${ }^{1}$, Mark Eilers ${ }^{2}$ \\ Learning and Cognitive Systems / Transportation Systems \\ C.v.O University / OFFIS, Oldenburg, Germany \\ http://www.lks.uni-oldenburg.de/ \\ claus.moebus@uni-oldenburg.de,mark.eilers@offis.de
}

\section{ABSTRACT}

Traffic scenario simulations and risk-based design require Digital Human Models (DHMs) of human control strategies. Furthermore, it is tempting to prototype assistance systems on the basis of a human driver model cloning an expert driver. We present the model architecture for embedding probabilistic models of human driver expertise with sharing of behaviors in different driving maneuvers. These models implement the sensory-motor system of human drivers in a mixtureof-behaviors (MoB) architecture with autonomous and goal-based attention allocation processes. A Bayesian MoB model is able to decompose complex skills (maneuvers) into basic skills (behaviors) and vice versa. The Bayesian-MoB-Model defines a probability distribution over driver-vehicle trajectories so that it has the ability to predict agent's behavior, to abduct hazardous situations, to generate anticipatory plans and control, and to plan counteractive measures by simulating counterfactual behaviors or actions preventing hazardous situations.

Keywords: Bayesian models of human driver behavior and cognition, probabilistic

${ }^{1}$ project Integrated Modeling for Safe Transportation (IMOST) sponsored by the Government of Lower Saxony, Germany under contracts ZN2245, ZN2253, ZN2366

${ }^{2}$ project ISi-PADAS funded by the European Commission in the 7 th Framework Program, Theme 7 Transport FP7-218552 
MÖBUS, C. \& EILERS, M., Mixture of Behaviors and Levels-of-Expertise in a Bayesian Autonomous Driver Model (paper accepted), 1st Intern. Conf. On Applied Digital Human Modeling, 17-20 July, 2010, Intercontinental, Miami Florida, USA

driver model, Bayesian autonomous driver models, mixture-of-behavior model, visual attention allocation, prediction and abduction of behavior, anticipatory plans and control, counteractive measures, risk and hazardous prevention

\section{INTRODUCTION}

The Human or Cognitive Centered Design (HCD) of intelligent transport systems requires digital Models of Human Behavior and Cognition (MHBC) which are embedded, context aware, personalized, adaptive, and anticipatory. A special kind of MHBC is the driver model which is used mainly in traffic scenario simulations and risk-based design (Cacciabue, 2007).

Modeling drivers is a challenging topic because no well established psychological theory about driving is at hand. Even simple maneuvers like braking are not well understood empirically. With the need for smarter assistance the problem of transferring human skills $(\mathrm{Xu}, 2005)$ without having a well-founded skill theory becomes more and more apparent.

The conventional approach for driver modeling is the handcrafting of MHBC. An ex post evaluation of their human likeness or empirical validity and revisionevaluation cycles is obligatory. We propose as a machine-learning alternative the estimation of Bayesian MHBCs from human behavior traces. The learnt models are empirical valid by construction. An ex post evaluation of Bayesian Autonomous Driver $(B A D)$ models is in principle not necessary when the statistical relations and conditional independencies between the pertinent variables in the data are mapped into the model.

The advantage of probabilistic models is their robustness facing the irreducible incompleteness of knowledge about the environment and the underlying psychological mechanisms (Bessiere, 2008).

\section{A BAYESIAN MIXTURE OF BEHAVIORS MODEL}

\section{BAYESIAN AUTONOMOUS DRIVER MODELS}

BAD models (Möbus et al., 2008; 2009a; 2009b, 2009c) are developed in the tradition of Bayesian expert systems (Pearl, 2009) and Bayesian (robot) Programming (Lebeltel et al., 2004, Bessiere et al., 2003, 2008). They describe phenomena on the basis of the joint probability distribution (JPD) and their factorization into conditional probability distributions (CPDs) of the observable pertinent variables. This is in contrast to models in cognitive architectures (e.g. ACT-R) which try to simulate latent or hidden cognitive algorithms and processes on a finer granular basis.

A BAD Mixture-of-Behaviors (BAD-MoB) model is a Bayesian Program (BP), which is able to decompose complex skills (scenarios, maneuvers) into basic skills 
MÖBUS, C. \& EILERS, M., Mixture of Behaviors and Levels-of-Expertise in a Bayesian Autonomous Driver Model (paper accepted), 1st Intern. Conf. On Applied Digital Human Modeling, 17-20 July, 2010, Intercontinental, Miami Florida, USA

(= behaviors, actions) and vice versa. The basic behaviors or sensory-motor schemas could be shared and reused in different maneuvers. Context dependent complex driver behavior will be generated by mixing the pure basic behaviors. The BAD-MoB-Model is embedded in a dynamic Bayesian network (DBN). If its template (Fig. 5) is rolled out (Fig. 6,7) it defines a probability distribution over driver-vehicle trajectories so that it has the ability to predict agent's behavior, to abduct hazardous situations (what could have been the initial situation), to generate anticipatory plans and control, and to plan counteractive measures by simulating counterfactual behaviors or actions preventing hazardous situations.

\section{BAYESIAN PROGRAMS AND DESCRIPTION COMBINATION}

A $B P$ is defined as a mean of specifying a family of probability distributions (Bessiere et al., 2003, 2008; Lebeltel et al., 2004). On the basis of a BP it is possible to construct a BAD-MoB-model, which can effectively control a (virtual) vehicle.

As Bessiere (2008) points out it is possible to combine or select single descriptions (= BPs) by a probabilistic if-then-else. "Description combination appears to implement naturally a mechanism similar to Hierarchical Mixture of Experts (Jordan and Jacobs, 1994) and is also closely related to mixture models ... From a programming point of view, description combination can be seen as a probabilistic if-then-else construction. $\mathrm{H}$ is the condition. If $\mathrm{H}$ is known with certainty, then we have a normal branching structure. If $\mathrm{H}$ is known with some uncertainty through a probability distribution, then the two possible consequences are automatically combined using weights proportional to this distribution." (Bessiere, 2008).

We embedded description combination (Lebeltel et al., 1999, 2004; Bessiere et al. 2003) in our DBN-based BAD-MoB-model. The condition variable $\mathrm{H}$ is a generalized case-statement like a Lisp cond and one of the root variables in our template model (Fig. 5), especially the variable Behaviors. The marginal probability distribution $\mathrm{P}\left(\right.$ Behaviors $\left.^{0}\right)$ or $\mathrm{P}\left(\right.$ Behaviors $\left.^{\mathrm{t}-1}\right)$ corresponds to the weighting or mixing coefficients of the description combination. The number of CPDs P(Action $\mid$ behavior, States, Percepts) equals to the cardinality Behaviors variable. For each behavior we have to establish a local CPD P(Action | behavior, States, Percepts). The collection of these local CPDs is the envisioned behavior library summarized in the total CPD P(Action | Behaviors, States, Percepts).

\section{LEVELS OF EXPERTISE AND MIXTURES OF BEHAVIORS}

BAD-MoB-models are dynamic Bayesian networks (DBNs) which can be considered as a subtype of a Bayesian Program (BP) (Bessiere, 2003). Under the assumption of stationarity their template models are specified as 2-time-slice Bayesian networks (2-TBNs). The template model can be unrolled so that their interface variables (Koller and Friedman, 2009) Behaviors and State are glued together producing an unrolled DBN over T time slices (T-TBN) like the 3-TBN in 
MÖBUS, C. \& EILERS, M., Mixture of Behaviors and Levels-of-Expertise in a Bayesian Autonomous Driver Model (paper accepted), 1st Intern. Conf. On Applied Digital Human Modeling, 17-20 July, 2010, Intercontinental, Miami Florida, USA

Fig. 6, 7. Learning data are time series of the pertinent domain-specific variables goals, behaviors, actions, observable states, and actions combined with posthoc annotations of maneuvers and scenarios. Information can be propagated within the T-TBN in various directions. When working top-down, goals emitted by higher cognitive layers of the agent activate a corresponding behavior which propagates actions, relevant areas of interest (AoIs), and expected perceptions. When working bottom-up, percepts trigger AoIs, actions, behaviors, and goals. When the task or goal is defined and there are percepts, evidence can be propagated simultaneously top-down and bottom-up, and the appropriate behavior can be activated.

Our DBN-based MoB model is influenced by the visual attention allocation model of Horrey et al. (2006) and the Bayesian filter and action selection model of Koike (2008). The BAD-MoB-model we present here is tailored to a virtual highway scenario assuming a hierarchy of driving skills or expertise.

\section{A Virtual Highway Scenario}

For the proof of concept we developed a 2-TBN for a simple scenario with three areas of interest (AoIs) and maneuvers (Fig. 1-3) (Möbus, et al., 2009c). The driver is sitting in the ego vehicle (ev). Sometimes an alter vehicle (av) or the roadside is occupying the AoIs depending on the state of the car (State $=$ left, middle, or right lane).
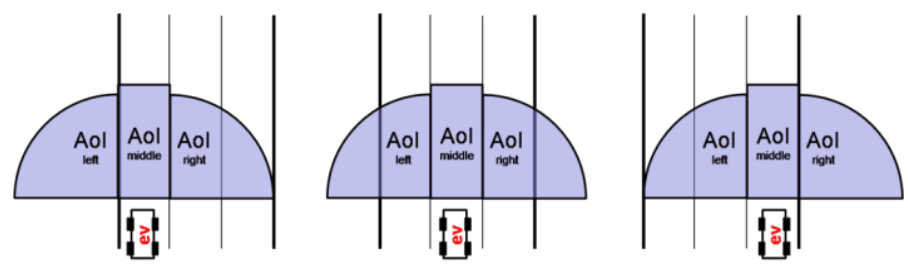

FIGURE 1. Areas of Interest (Aols) and Ego Vehicle Positions
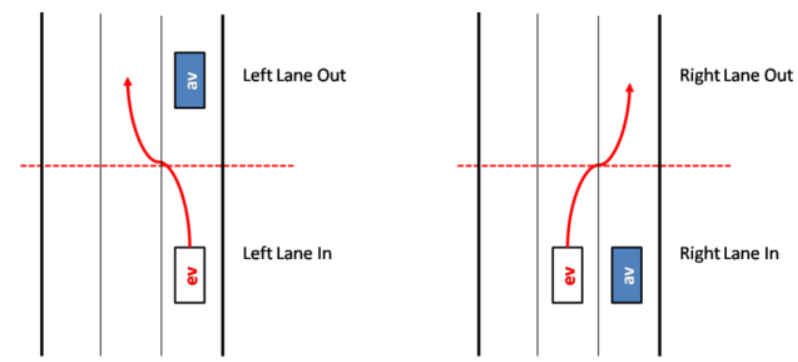

driving actions with focus on the left: left check lane, left signal, left turn

driving actions with focus in the middle: acceleration, deceleration, look forward

driving actions with focus on the right : right check lane right signal, right turn

FIGURE 2. Driving Maneuvers LeftLaneChange LLC (left) and RightLaneChange RLC (right) with two sequences of Driving Behaviors each (above, below) 
MÖBUS, C. \& EILERS, M., Mixture of Behaviors and Levels-of-Expertise in a Bayesian Autonomous Driver Model (paper accepted), 1st Intern. Conf. On Applied Digital Human Modeling, 17-20 July, 2010, Intercontinental, Miami Florida, USA

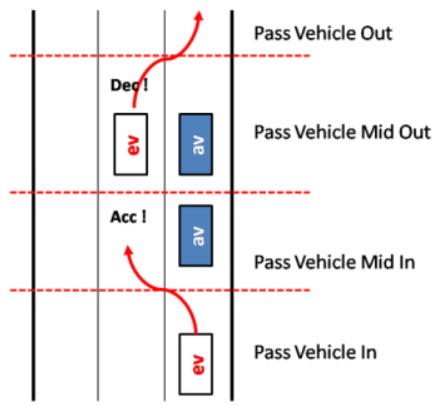

driving actions with focus on the left:

left check lane, left signal, left turn

driving actions

with focus in the middle:

acceleration, deceleration, look forward

driving actions

with focus on the right:

right check lane right signal, right turn

FIGURE 3 Pass Vehicle Driving Maneuver with a sequence of 4 Driving Behaviors

The levels of expertise, the model components (layer, sequence) and a partial grammar of expertise are shown in Fig. 4.

\begin{tabular}{|l|l|l|}
\hline $\begin{array}{l}\text { Levels of } \\
\text { Expertise }\end{array}$ & \multicolumn{1}{|c|}{$\begin{array}{c}\text { Model } \\
\text { Component }\end{array}$} & \multicolumn{1}{c|}{ Hierarchy of Skills, Levels of Expertise } \\
\hline Skills & & Skills $=\{\ldots$, drivingScenarioSkills, ... $\}$ \\
\hline $\begin{array}{l}\text { Scenario } \\
\text { Skills }\end{array}$ & & $\begin{array}{l}\text { DrivingScenarioSkills } \\
=\{\text { highway, countryRoad, city }\}\end{array}$ \\
\hline $\begin{array}{l}\text { Driving } \\
\text { Maneuver } \\
\text { Skills }\end{array}$ & $\begin{array}{l}\text { Driving Maneuver } \\
\text { Sequence } \\
\text { (horizontally } \\
\text { distributed) }\end{array}$ & $\begin{array}{l}\text { highway.Maneuvers } \\
=\{\text { leftLaneChange (ILC), rightLaneChange (rLC), passVehicle } \\
\text { (pV), newManeuver }\}\end{array}$ \\
\hline $\begin{array}{l}\text { Driving } \\
\text { Behavior } \\
\text { Skills }\end{array}$ & $\begin{array}{l}\text { Driving Behavior } \\
\text { Layer }\end{array}$ & $\begin{array}{l}\text { Behaviors } \\
=\{\text { leftLaneln (ILI), leftLaneOut (ILO), passin (pl), passMidln } \\
\text { (pMI), passMidOut (pMO), passOut (pO), rightLaneln (rLI), } \\
\text { rightLaneOut (rO), newBehavior }\} \\
\text { e.g.: leftLaneChange.Behaviors = \{leftLaneln, leftLaneOut }\}\end{array}$ \\
\hline $\begin{array}{l}\text { Driving } \\
\text { Action } \\
\text { Skills }\end{array}$ & $\begin{array}{l}\text { Driving Actions } \\
\text { Layer }\end{array}$ & $\begin{array}{l}\text { Actions } \\
=\{\text { leftCheckLane (ICL), leftSignal (IS), leftTurn (IT), } \\
\text { middleAcceleration (mA), middleDeceleration (mD), } \\
\text { middleLookForward (mLF), rightCheckLane (rCL), rightSignal } \\
\text { (rS), rightTurn (rT) }\} \\
\text { e.g.: leftLaneln.Actions = \{lCL, mD, mLF, IS, IT, mA }\}\end{array}$ \\
\hline
\end{tabular}

FIGURE 4 Levels of Expertise, Model Components, and part of Expertise Grammar

\section{Dynamic Reactive BAD-MoB-model}

For our BAD-MoB-model we propose partially inverted dynamic Bayesian networks (DBNs) of the 2-TBN-type (Fig. 5). We call the model Dynamic Reactive $M o B$ Model. The model is reactive because AoIs directly influence actions. The model embeds two naïve Bayesian classifiers: One for the Behaviors and one for the States. This simplifies the structure of the architecture. Time slices are selected so that in each new time slice a new behavior is active. A sequence of behaviors implements a single maneuver. When we replace the reactive submodel for the Actions variable in Fig. 5 by a third classifier we can simplify the model and reduce the number of parameters by $79 \%$. 
MÖBUS, C. \& EILERS, M., Mixture of Behaviors and Levels-of-Expertise in a Bayesian Autonomous Driver Model (paper accepted), 1st Intern. Conf. On Applied Digital Human Modeling, 17-20 July, 2010, Intercontinental, Miami Florida, USA

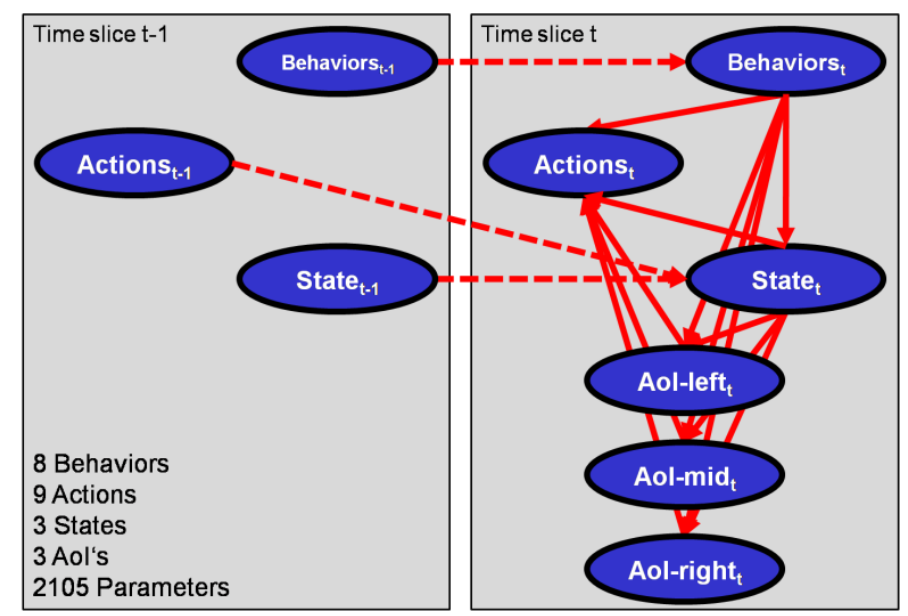

FIGURE 5 Dynamic Reactive BAD-MoB-model with Behavior and State Classifiers

The top layer consists of behavior nodes. There are behaviors for each main part of a maneuver (Fig. 2-4): left_lane_in,.... The next layer describes the actions the model is able to generate: left_check_lane,.... Below that appears the node state of the vehicle $\left(i s \_i n \_l e f t\right.$ lane, ... ). Then there are three bottom layers contain nodes for the three AoIs with values is_occupied and is_empty.

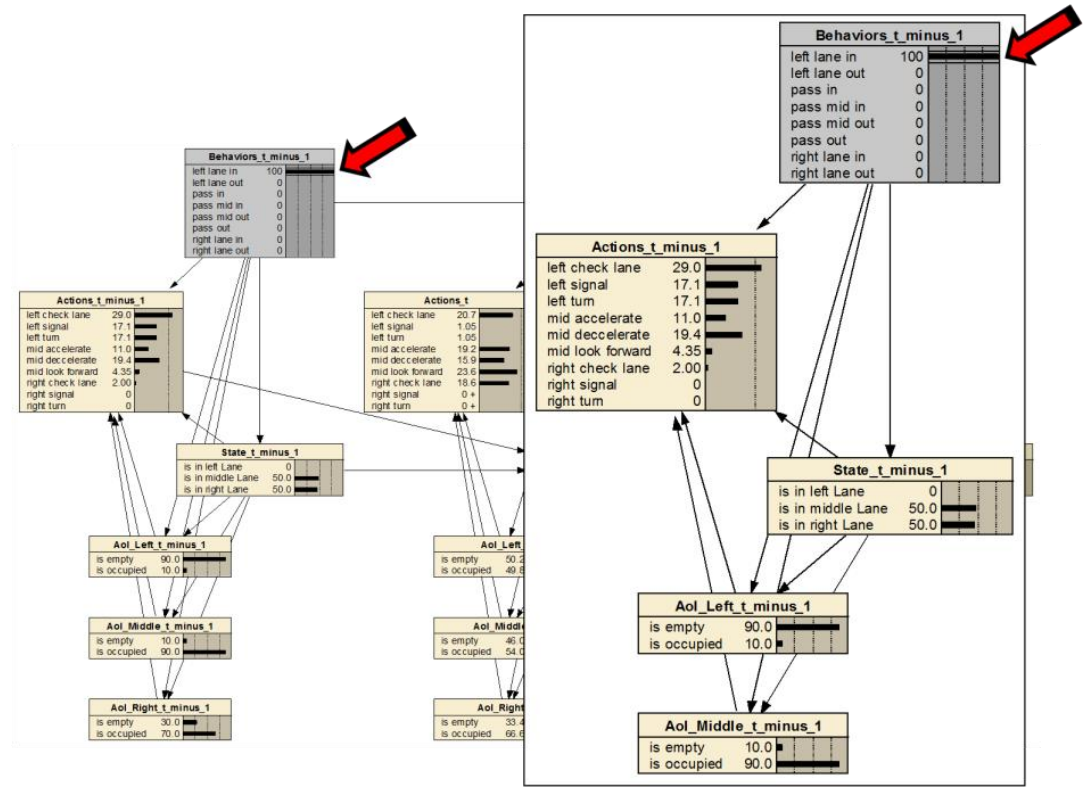

FIGURE 6 Expected behavior of the 3-TBN model with the goal behavior left_lane_in (the left-upper part of time-slice $\mathrm{t}-1$ is expanded on the right of the figure) 
MÖBUS, C. \& EILERS, M., Mixture of Behaviors and Levels-of-Expertise in a Bayesian Autonomous Driver Model (paper accepted), 1st Intern. Conf. On Applied Digital Human Modeling, 17-20 July, 2010, Intercontinental, Miami Florida, USA

An implementation in NETICA with artificial but plausible data is shown in Fig 6. When the model is urged to be in the left_lane_in behavior by e.g. goal setting from a higher cognitive layer, we expect in the same time-slice primarily that the left lane is checked and that the driver decelerates the vehicle. For the AoIs we expect that the middle AoI is occupied and the left AoI is empty. For the this time slice we expect the vehicle in the right or middle lane. The expected behavior changes between the time-slices. So the expected behavior in time-slice $t$ is the left_lane_out behavior. We have higher beliefs in acceleration, attention forward and in checking the left and right lane.

When the state is known (e.g. State $\left.=i s \_i n \_m i d d l e \_l a n e\right)$ we can include this as a single evidence in the model and infer the appropriate expectations (e.g. left and right lane check, looking forward, and both (ac|de)celerations).

When the model perceives a combination of AoI evidence, we can infer the behaviors. For instance, when the left AoI is empty and the middle and right AoI is occupied. We expect that the vehicle is in the middle or right lane, that the behaviors left_lane_in and pass_in are ambiguous, and that their appropriate mixed behavior (left_lane_check, deceleration) is activated. In the case, when all AoIs are occupied the model is decelerating with main attention to the middle AoI (middle_straight_look).

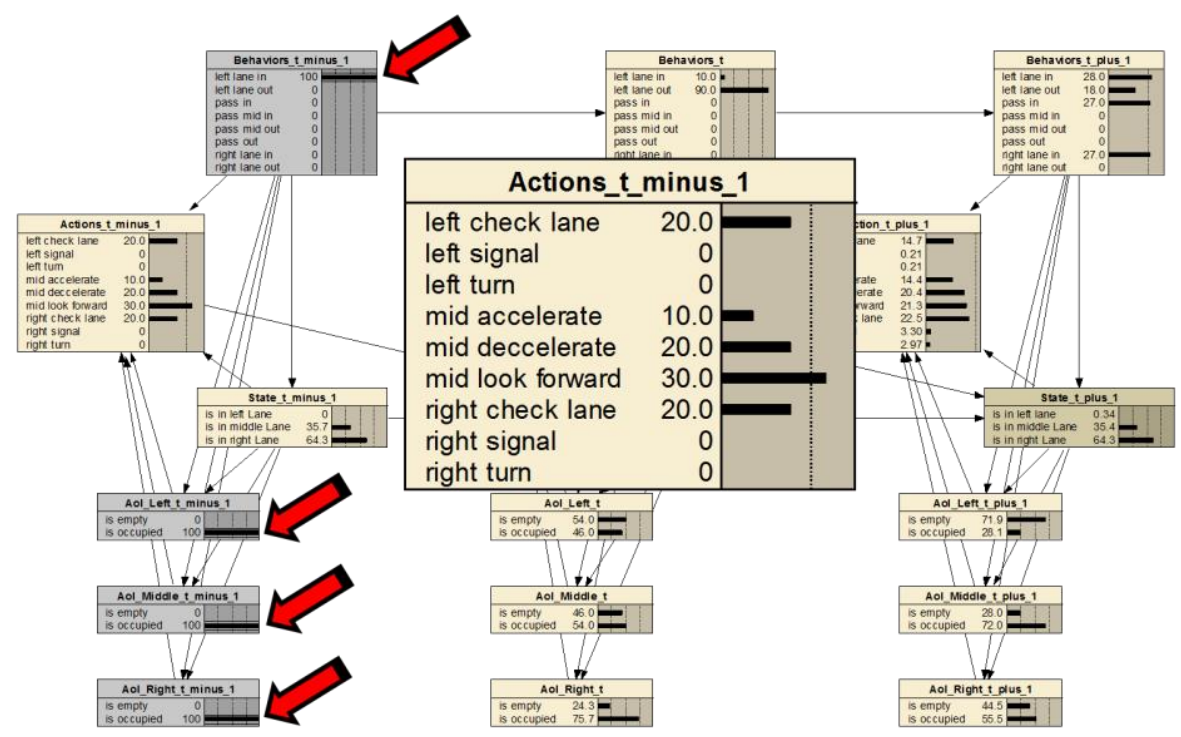

FIGURE 7 Conditional distributions in Dynamic Reactive MoB Model when receiving a combination of Behavior (goal) and blocking Aol evidence (Action-node expanded)

What will happen, if a goal is blocked? In Fig. 7 this is modeled by the appropriate evidence. The lane-in behavior is activated as a goal and at the same time the perception in the left and middle AoIs is set to is_occupied. This situation blocks the left lane in and the pass vehicle in behaviors. The expected actions are 
MÖBUS, C. \& EILERS, M., Mixture of Behaviors and Levels-of-Expertise in a Bayesian Autonomous Driver Model (paper accepted), 1st Intern. Conf. On Applied Digital Human Modeling, 17-20 July, 2010, Intercontinental, Miami Florida, USA

looking forward, checking left and right lanes, and deceleration. These are typical behavioral indicators for helplessness and stress.

This architecture has the ability to predict agent's behavior, to abduct hazardous situations (what could have been the initial situation), to generate anticipatory plans and control, and to plan counteractive measures by simulating counterfactual behaviors or actions preventing hazardous situations. For these applications we have to provide the model with appropriate evidence and questions.

For instance when planning counteractive measures by simulating counterfactual behaviors or actions preventing hazardous situations we need a 3step procedure (Pearl, 2009): (1) abduction of a hazardous situation backwards with the full state-based BAD-MoB-model, (2) mutilate the full model to a reduced model, that is able to predict intervention effects, (3) experiment with counterfactual actions (= countermeasures) by providing action evidence in the reduced model and predicting the action effects.

\section{FIRST MODELING RESULTS WITH REAL DATA}

BAD-models with Mixed Behaviors are expressive enough to describe and predict a wide range of phenomena. In Möbus \& Eilers (2009a) we presented a BAD model for lateral and longitudinal control without behavior mixing. The model showed nearly perfect behavior on the Aalborg course in the racing simulation game TORCS, though some suboptimal driving maneuvers could be observed. This is due to the fact that we used a fixed set of parameters in our model on a track with different segments like hair-needle curve, straight line segments etc. We modified the BAD-model architecture introducing concepts of the theory of ambient vision (Horrey et al., 2006). This led to a slightly simplified version of a BAD-MoB-model with two behavior and steer-action classifiers (Fig. 8).

The results are very promising as can be seen from Figs. 9 and 10. In Figure 9 the driver is driving in a right bended curve. His ambient vision field is sampled by 20 sensors (Fig. 9, left). Provided this perceptional evidence the conditional distribution for the action variable Steer (= steering angle) and the behavior variable Behaviors (= Experts) are inferred (Fig. 9, middle, right). As can be seen only the right-turn behavior (expert) is recognized and the corresponding angle of the steering-action is inferred. Sampling a concrete steering action from this conditional probability distribution gives the generated action of the BAD-MoB-model. Leaving the right-bended curve (Fig. 10) activates actions which are a mixture of the two behaviors (experts) straight and right (Fig. 10, right).

\section{CONCLUSION AND OUTLOOK}

We demonstrated that the DBN-based BAD-MoB-model has the ability to predict agent's behavior, to abduct hazardous situations (what could have been the 
MÖBUS, C. \& EILERS, M., Mixture of Behaviors and Levels-of-Expertise in a Bayesian Autonomous Driver Model (paper accepted), 1st Intern. Conf. On Applied Digital Human Modeling, 17-20 July, 2010, Intercontinental, Miami Florida, USA

initial situation), to generate anticipatory plans and control, and to plan counteractive measures by simulating counterfactual behaviors or actions preventing hazardous situations. In Eilers and Möbus (2010) we present an efficient implementation. The next research steps will work on the vertical refinement of models interfacing single actions with more concrete behaviors.

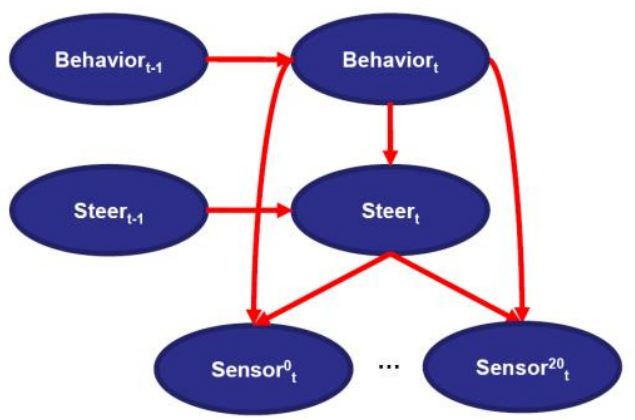

FIGURE 8 Dynamic BAD-MoB-model with Bayesian Classifiers Behavior and Steer
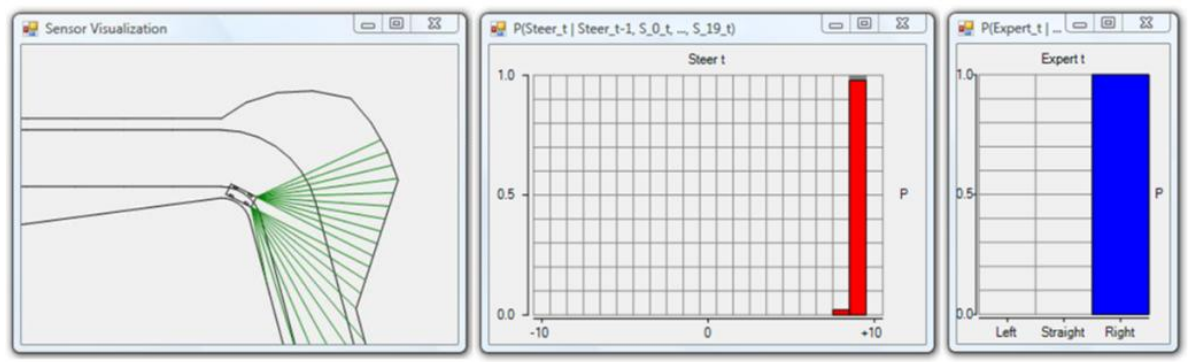

Fig. 9 Ambient perceptional evidence (left) and conditional distributions (middle, right) in Dynamic Partial Inverted BAD-MoB-model with 2 Bayesian Classifiers when driving in a right bended curve
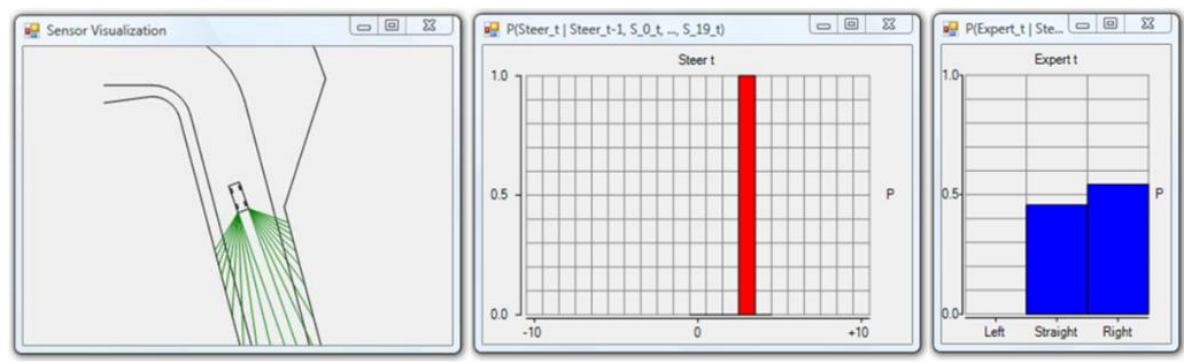

Fig. 10 Ambient perceptional evidence (left) and conditional distributions (middle, right) in Dynamic Partial Inverted BAD-MoB-model with 2 Bayesian Classifiers when leaving a right bended curve 
MÖBUS, C. \& EILERS, M., Mixture of Behaviors and Levels-of-Expertise in a Bayesian Autonomous Driver Model (paper accepted), 1st Intern. Conf. On Applied Digital Human Modeling, 17-20 July, 2010, Intercontinental, Miami Florida, USA

\section{REFERENCES}

Bessiere, P. (2003). Survey: Probabilistic Methodology and Techniques for Artifact Conception and Development, Rapport de Recherche, No. 4730, INRIA

Bessiere, P. Laugier, Ch. and Siegwart, R. (eds.) (2008). Probabilistic Reasoning and Decision Making in Sensory-Motor Systems, Berlin: Springer, ISBN 978-3-540-79006-8

Cacciabue, P.C. (ed). (2007). Modeling Driver Behaviour in Automotive Environments, London: Springer, ISBN-10: 1-84628-617-4

Eilers, M. and Möbus, C. (2010). Learning of a Bayesian Autonomous Driver Mixture-ofBehaviors (BAD MoB) Model, (this proceedings), invited Special Interest Group: Möbus, C. \& Bessiere, P., Models of Human Behavior and Cognition in the Bayesian Programming Framework, 1st International Conference On Applied Digital Human Modeling, 17-20 July, 2010, Intercontinental, Miami Florida, USA

Horrey, W.J. et al. (2006). Modeling Driver's Visual Attention Allocation While Interacting With In-Vehicle Technologies, J. Exp. Psych., 12, 67-78

Koike, C.C. Bessiere, P., and Mazer, E. (2008). Bayesian Approach to Action Selection and Attention Focusing, in Bessiere et al., (Eds.), Probabilistic Reasoning and Decision Making in Sensory-Motor Systems, Berlin: Springer, 177- 201

Koller, D., Friedman, N. (2009). Probabilistic Graphical Models, Cambridge, Mass.: MIT Press, ISBN 978-0-262-01319-2

Lebeltel, O. Bessiere, P. Diard, J. and E. Mazer (2004). Bayesian Robot Programming, Advanced Robotics, 16 (1), 49-79

Möbus, C. and Eilers, M. (2008). First Steps Towards Driver Modeling According to the Bayesian Programming Approach, Symposium Cognitive Modeling, p.59, in: L. Urbas, Th. Goschke \& B. Velichkovsky (eds) KogWis 2008. Christoph Hille, Dresden, ISBN 978-3-939025-14-6

Möbus, C. Eilers, M. (2009a). Further Steps Towards Driver Modeling according to the Bayesian Programming Approach, in: Conference Proceedings, HCII 2009, Digital Human Modeling, pp. 413-422, LNCS (LNAI), Springer, San Diego, ISBN 978-3-64202808-3

Möbus, C. Eilers, M. Garbe, H., and Zilinski, M. (2009b). Probabilistic and Empirical Grounded Modeling of Agents in (Partial) Cooperative Traffic Scenarios, in: Conference Proceedings, HCII 2009, Digital Human Modeling, pp. 423-432, LNCS (LNAI), Springer, San Diego, ISBN 978-3-642-02808-3

Möbus, C. Eilers, M. Zilinski, M. Garbe, H. (2009c). Mixture of Behaviors in a Bayesian Driver Model, in: Lichtenstein, A. et al. (eds), Der Mensch im Mittelpunkt technischer Systeme, p.96 and p.221-226 (CD), Düsseldorf: VDI Verlag, ISBN 978-3-18-302922-8, ISSN 1439-958X

Pearl, J. (2009). Causality - Models, Reasoning, and Inference, 2nd ed., Cambridge University Press, ISBN 978-0-521-89560-6

Yangsheng $\mathrm{Xu}, \mathrm{Ka}$ Keung Caramon Lee, and Ka Keung C. Lee, Human Behavior Learning and Transfer, CRC Press Inc., (2005) 